\title{
Genomic resources for a unique, low- virulence Babesia taxon from China
}

\author{
Guiquan Guan ${ }^{1,2+}$, Pasi K. Korhonen ${ }^{2 \dagger}$, Neil D. Young ${ }^{2}$, Anson V. Koehler ${ }^{2}$, Tao Wang ${ }^{2}$, Youquan Li', Zhijie Liu', \\ Jianxun Luo ${ }^{1}$, Hong Yin ${ }^{1 *}$ and Robin B. Gasser ${ }^{2^{*}}$
}

\begin{abstract}
Background: Babesiosis is a socioeconomically important tick-borne disease of animals (including humans) caused by haemoprotozoan parasites. The severity of babesiosis relates to host and parasite factors, particularly virulence/ pathogenicity. Although Babesia bovis is a particularly pathogenic species of cattle, there are species of Babesia of ruminants that have limited pathogenicity. For instance, the operational taxonomic unit Babesia sp. Xinjiang (abbreviated here as $B x$ ) of sheep from China is substantially less virulent/pathogenic than B. bovis is in cattle. Although the reason for this distinctiveness is presently unknown, it is possible that $B x$ has a reduced ability to adhere to cells or evade/suppress immune responses, which might relate to particular proteins, such as the variant erythrocyte surface antigens (VESAs).

Results: We sequenced and annotated the $8.4 \mathrm{Mb}$ nuclear draft genome of $B x$ and compared it with those of $B$. bovis and B. bigemina by synteny analysis; we also investigated the genetic relationship of $B x$ with selected Babesia species and related apicomplexans for which genomic datasets are available, and explored the VESA complement in $B x$.
\end{abstract}

Conclusions: The availability of the $B x$ genome now provides unique opportunities to elucidate aspects of the molecular biology, biochemistry and physiology of $B x$, and to explore the reason(s) for its limited virulence and/or apparent ability to evade immune attack by the host animal. Moreover, the present genomic resource and an in vitro culture system for Bx raises the prospect of establishing a functional genomic platform to explore essential genes as new intervention targets against babesiosis.

Keywords: Babesia sp. Xinjiang, China, Sheep, Genome, Variant erythrocyte surface antigens (VESAs)

\section{Background}

Babesiosis is a globally important tick-borne, parasitic disease of animals, including humans, caused by haemoprotozoans of the genus Babesia (phylum Apicomplexa). This disease has a major, adverse economic impact on the health and productivity of livestock animals, particularly ruminants, as a consequence death, reduced meat and milk production, increased sterility and abortion rates and/or the cost of treatment and prevention [1], and is an ongoing problem particularly in tropical and

\footnotetext{
*Correspondence: yinhong@caas.cn; robinbg@unimelb.edu.au

${ }^{\dagger}$ Equal contributors

'State Key Laboratory of Veterinary Etiological Biology, Lanzhou Veterinary Research Institute, Chinese Academy of Agricultural Sciences, Lanzhou, Gansu, China

${ }^{2}$ Faculty of Veterinary and Agricultural Sciences, The University of Melbourne, Parkville, VIC 3010, Australia
}

(c) The Author(s). 2016 Open Access This article is distributed under the terms of the Creative Commons Attribution 4.0 International License (http://creativecommons.org/licenses/by/4.0/), which permits unrestricted use, distribution, and reproduction in any medium, provided you give appropriate credit to the original author(s) and the source, provide a link to the Creative Commons license, and indicate if changes were made. The Creative Commons Public Domain Dedication waiver (http://creativecommons.org/publicdomain/zero/1.0/) applies to the data made available in this article, unless otherwise stated.

subtropical regions of Australia, Africa and the Americas. Most economic impact appears to be linked to bovine babesiosis, caused by Babesia bovis and B. bigemina, but the socioeconomic importance of babesiosis in small ruminants is also likely to be considerable in some countries $[2,3]$.

Babesia spp. are transmitted to their mammalian hosts by particular ixodid tick species. The tick injects sporozoites into the blood stream upon feeding; these 'zoites directly invade the erythrocyte and undergo asexual replication (binary fission) to produce many merozoites that are released into the circulation following erythrocyte rupture and then reinvade erythrocytes, and the cycle continues. This rapid, perpetual cycle of replication (merogony) and associated erythrocyte invasion and destruction usually lead to intravascular haemolysis, anaemia, haemoglobinuria and/or jaundice. The severity 
of disease usually relates to host and parasite factors, but often the virulence/pathogenicity is of considerable importance. For example, B. bovis is particularly pathogenic in Bos taurus and can dramatically modify the structure and functionality of infected erythrocytes [4, 5]; this alteration can be accompanied by an accumulation of affected erythrocytes in the capillaries of organs, including the brain and lungs, leading to severe cerebral disease, respiratory insufficiency and/or multi-organ failure. Interestingly, in contrast to B. bovis, there are species of Babesia of ruminants that have limited pathogenicity. For example, the operational taxonomic unit Babesia sp. Xinjiang (abbreviated as $B x$ ) of sheep from central and northwestern regions of China, which is transmitted by Hyalomma anatolicum anatolicum, has been reported to have limited virulence/pathogenicity in sheep (Ovis aries) [6, 7]. Although the reason/s for this observation is/are not yet known, it is possible that $B x$ has a reduced ability to adhere to cells or evade/suppress immune responses, which might relate to particular protein groups, including variant erythrocyte surface antigens (VESAs) and/or small open reading frame (SmORF) proteins [8].

The availability of an effective and continuous in vitroculture system for $B x$ [9] provides unique opportunities for detailed investigations of antigenic variation, virulence factors, the parasites' biology and molecular biology via, for instance, functional genomics [5], with a future prospect of discovering new intervention methods against babesiosis more generally. To provide a foundation for such research areas, in the present study, we (i) sequenced the nuclear genome of $B x$ and compared its first draft genome with those of B. bovis and B. bigemina by synteny analysis; (ii) studied the genetic relationship of $B x$ with other Babesia species and related apicomplexans for which genomic datasets are available; and (iii) explored the complement of ves genes and their predicted proteins in $B x$.

\section{Methods}

\section{Sequencing and preparation of data}

Merozoites of Babesia sp. Xinjiang [7] were maintained in sheep erythrocytes in a continuous in vitro culture and amplified in a parasite-free, splenectomised sheep [9]. Merozoites were purified from blood as described [10], and genomic DNA was isolated using the Gentra Puregene kit (Qiagen, Hilden, Germany) and total RNA employing TriPure (Sigma-Aldrich, St Louis, MI, USA), according to the manufacturers' protocols. The nucleic acids were quantitated using a fluorometer (Qubit, Invitrogen, Carlsbad, CA, USA), and their quality was verified using a BioAnalyzer (2100, Agilent). One pairedend (500 bp insert size) and two mate-pair ( $2 \mathrm{~kb}$ and $5 \mathrm{~kb})$ genomic DNA libraries were sequenced using
Illumina technology (HiSeq; $2 \times 100$ reads for paired-end libraries, and $2 \times 49$ reads for mate-pair libraries), and RNA-sequencing (RNA-seq) was conducted using an established protocol (Illumina). Genomic and RNA-seq reads were quality-filtered using the program Trimmomatic v.0.36 [11], and RNA-seq reads were processed further using Khmer v.2.0 [12].

\section{Prediction of repetitive elements}

First, genomic repeats were modelled using the program RepeatModeler [13], and repeat predictions merged using the programs RECON [14], RepeatScout [15] and Tandem Repeat Finder (TRF) [16]. Second, long terminal repeats (LTRs) were predicted using the program LTR_Finder [17]. Third, simple repeats and transposons were predicted using RepeatMasker v.4.0.5 [18], with transposons being predicted using data from Repbase v.17.02 [19]. Fourth, all repeats were combined using RepeatMasker v4.0.5.

\section{Genomic assembly and gene prediction}

Short-read data were assembled using the program SPAdes v3.5.0 [20] and scaffolded using the program SSPACE v3.0 [21]. Genes were predicted with MAKER2 [22] using the msoftware suite containing the $a b$ initio-gene prediction programs AUGUSTUS [23], GeneMark-ES [24] and SNAP [25]. Genome-guided gene predictions using RNA-seq read data were conducted using TopHat2 v2.1.0 [26] and Cufflinks2 v2.2.1 [27]. RNA data were assembled using both de novo- and genome-guided approaches using the Trinity platform [28]. The resultant transcriptome, together with a set of proteomes from NCBI protein database [29] for B. bovis, B. microti, Cryptosporidium hominis, C. muris, C. parvum, Neospora caninum, Plasmodium falciparum, Theileria annulata, Th. parva, Toxoplasma gondii and Tetrahymena thermophila, was used as 'evidence data' for gene prediction. EVidenceModeler (EVM) software [30] was utilised to combine gene predictions as well as protein sequence and transcript alignments into weighted consensus gene structures. In short, the transcriptomic data set was mapped to the genome using the pipeline PASA2 [30]; the resultant gene predictions, transcriptome and proteome mappings from the MAKER2 prediction were then integrated using EVM. The resultant protein-coding gene set was then consolidated using the following approach: (i) genes containing repeats that overlapped by $\geq 80 \%$, had $\leq 20 \%$ transcript support and whose codon usage was consistent with a coding region, as established using program TransDecoder (within the Trinity), were removed; and (ii) genes containing repeats that overlapped by $\geq 80 \%$ or had $\leq 20 \%$ transcript support and whose codon usage was not consistent with a coding region were removed. Finally, the tRNA genes were predicted using the program tRNAscan-SE [31]. For the predicted genes, 
the genome completeness was estimated using the program Benchmarking Universal Single Copy Orthologs (BUSCO) [32].

\section{Genome annotation}

Protein-coding genes were annotated using the programs InterPro [33] and BLAST+ [34, 35]. BLAST+ was applied to the proteome of $B$. bovis [36], and to the databases UniProtKB/SwissProt [37], KEGG [38] and NCBI protein $\mathrm{nr}$ [39]. Signal peptides were predicted using SignalP [40] and transmembrane protein regions employing TMHMM [41]. A custom script was created to convert the assembly, the predicted genes and the gene annotations into Abstract Syntax Notation One (ASN.1) for NCBI submission. The program Genome Annotation Generator (GAG) v1.0 [42] was used in this custom script.

\section{Phylogenetic analysis}

First, single copy orthologous (SCO) protein-coding genes shared by the proteomes of 16 species $(B x, B$. bovis, B. bigemina; C. parvum, C. hominis; Eimeria tenella; P. chabaudi, P. falciparum, P. knowlesi, P. vivax; Th. annulata, Th. equi, Th. orientalis, Th. parva; To. gondii; and Te. thermophila) were identified using the program OrthoMCL $[43,44]$. The 16 amino acid sequences representing individual SCOs were aligned using the program MAFFT v7.271 [45], and the SCOs with a minimum gap-free alignment length of 20 amino acids and with at least one phylogenetically informative site were selected. The final subset of SCOs common to all 16 species were then concatenated and subjected to phylogenetic analyses using the methods Bayesian inference (BI) in MrBayes v.3.2.2 [46, 47] and Maximum Likelihood (ML) in RAxML v.8.0.24 [48]; Te. thermophila was used as an outgroup. For BI, following the model selection using the program ProtTest 3.4 [49], the prior evolution model for amino acids was set to WAG [50], and the likelihood model was set to invgamma [51, 52]; from 200,000 Markov Chain Monte Carlo (MCMC) [53-55] iterations, the first 50,000 were discarded as non-converged burn-in, and nodal support values were given as posterior probabilities. For ML, the JTT [56], evolution model was used and the concatenated alignment blocks were bootstrapped 100 times to infer nodal support values. Phylogenetic trees were drawn using FigTree v1.4 (http://tree.bio.ed.ac.uk/software/figtree/).

\section{Synteny}

Synteny among $B x, B$. bovis and $B$. bigemina was established using a custom script. The scaffold-pairs containing SCOs shared between the two species were converted into a bipartite graph and processed in a one- sided crossing minimization algorithm [57] employing the program DSDP5 (a software for semi-definite programming) [58].

\section{Variant erythrocyte surface (ves) antigen genes}

These genes were first predicted using the program BLASTp (E-value $10^{-8}$ ) in the annotation of the draft genome for $B x$ and then using the HMM models for $B$. bovis, B. bigemina and B. divergens [8]. The predicted genes were then aligned using the program MAFFT, and shared amino acid patterns of encoded proteins identified manually and using the program PRATT v2.1 [59]. The program HMMER v3.1.2 [60] was used to search for the genes encoding proteins with a VESA1_N domain for variants $a$ and $b$, listed in the Pfam database [61]. The matching domain sequences were extracted from protein sequences using a custom script and aligned using MAFFT. A $B x$-specific VESA1_N HMM model was created using the program HMMER. This model was used to predict the VESA1_N domain in predicted proteins of $B x$. The ves genes encoding proteins with this domain as well as shared patterns were drawn using a custom script.

\section{Results and discussion}

The draft nuclear genome of $B x$ is $8.4 \mathrm{Mb}$ in size (Table 1). We detected 195 of 429 core essential genes by BUSCO, suggesting a near complete genome. The $B x$ genome is similar in size with the congeners $B$. bovis (8.2 $\mathrm{Mb})$, but smaller than $B$. bigemina $(13.8 \mathrm{Mb})$ and larger than B. microti $(6.5 \mathrm{Mb})[8,36,62]$. We estimated the repeat content of this draft genome at $4.3 \%$, equating to $365.6 \mathrm{~kb}$, of which interspersed repeats comprised 145 LINEs, 5 DNA and 431 unclassified elements.

We annotated and then compared the gene set of $B x$ with those of B. bovis, B. bigemina and B. microti as well as other selected apicomplexans. In the draft genome of $B x$, we identified 3066 protein-coding genes, 754 of which were supported by transcriptomic data for merozoites, with a mean total length of $1.96 \mathrm{~kb}$, mean exon length of $530 \mathrm{bp}$ and a mean of 3.3 exons per gene (see Table 1). Approximately $96.8 \%(n=2969)$ of the predicted $B x$ genes (Fig. 1) have an homolog (BLASTp cut-off: $10^{-8}$ ) in B. bovis (2874; $92.7 \%)$, B. bigemina (2907; $94.8 \%$ ) or B. microti (2227; $72.6 \%)[8,36,62]$. A total of $1960 B x$ genes are orthologous (OrthoMCL BLASTp cut-off $10^{-8}$ ) among all four taxa of Babesia, and 2894 were shared by at least one other taxon (Fig. 1). Conversely, 172 (5.6\%) genes are unique to $B x$ (Fig. 1). Of the entire $B x$ gene set, 984 genes had an ortho$\log \left(\leq 10^{-8}\right)$ linked to 246 known biological pathways (see Additional file 1). Comparison of universal SCOs among these four Babesia taxa (cf. Table 1; $n=195$ for $B x$ and $n=$ 204-212 for others) indicates that the majority of $B x$ genes are represented in the present genomic assembly for $B x$. 
Table 1 Features of the draft genome of Babesia sp. Xinjiang (Bx) with those of B. bovis, B. bigemina and B. microti

\begin{tabular}{|c|c|c|c|c|}
\hline Features & Babesia sp. Xinjiang $(B x)$ & Babesia bovis & Babesia bigemina & Babesia microt \\
\hline Genome size (Mb) & 8.4 & 8.2 & 13.8 & 6.5 \\
\hline Number of scaffolds or chromosomes & 215 & 4 & 6 & 3 \\
\hline N50 for scaffolds (kb) & 533.30 & - & 3520 & - \\
\hline N90 for scaffolds (kb) & 96.98 & - & - & - \\
\hline Genome GC content (\%) & 43.9 & 41.5 & 50.6 & 36.0 \\
\hline Repetitive sequences (\%) & 4.3 & - & - & - \\
\hline Exonic proportion/incl. introns (\%) & $63 / 71$ & $70 / 73$ & -163 & $73 / 81$ \\
\hline Number of nuclear protein-coding genes & 3066 & 3706 & 4457 & 3513 \\
\hline Gene density (bp per gene) & & 2194 & 2306 & 1816 \\
\hline Mean gene length including introns (bp) & 1958 & 1609 & 1531 & 1471 \\
\hline Mean CDS length (bp) & 1721 & 1503 & - & 1327 \\
\hline Mean exon number per gene & 3.3 & 2.8 & - & 3.3 \\
\hline Mean exon length (bp) & 530 & 547 & - & 397 \\
\hline Mean intron length (bp) & 106 & 60 & - & 61 \\
\hline Coding GC content (\%) & 45.4 & 44.0 & 51.7 & 39.0 \\
\hline Number of predicted tRNAs & 41 & 70 & - & 44 \\
\hline BUSCO completeness (\%/count) & $45 / 195$ & $48 / 204$ & $49 / 210$ & $49 / 212$ \\
\hline
\end{tabular}

As the specific status of $B x$ has not yet been resolved, we were keen to assess its evolutionary relationship with known Babesia species and other apicomplexan haematoprotozoa (Theileria and Plasmodium) for which published genomes were available. Based on two independent analyses of sequence data for 326 shared SCOs, we showed that $B x$ from sheep was more closely related to $B$. bovis than to B. bigemina from cattle, a finding supported by a genome-wide syntenic comparison among the three taxa (Fig. 1). Although the number of genome sequences publicly available for Babesia is presently limited, this finding is interesting, given the discrepancy in pathogenicity between $B x$ and $B$. bovis. Of note was also the result that species of Babesia and of Theileria each grouped together (and grouped with one another) to the exclusion of B. microti, suggesting that the latter species does not belong to either the genus Babesia or Theileria. This finding is supported by previous evidence from other phylogenetic analyses using data representing a small number of genetic markers (e.g. [63-65]) and 316 genes [62]. The present results show that Babesia is a paraphyletic group, indicating that the taxonomy of members of this genus needs to be revised; they also show that $B$. microti has diverged early during piroplasm evolution. Overall, these findings also suggest that $B$. microti represents a new genus that is distinct from both Babesia and Theileria, in accord with a previous proposal [62].

Although $B x$ appears to be closely related to $B$. bovis (cf. Fig. 1), there is a distinct difference between these two species in their pathogenicity in their respective host animals. As indicated, on one hand, $B x$ is virtually nonpathogenic in susceptible sheep (and not infective to calves or goats) [9], whereas B. bovis is highly pathogenic in the naïve bovine host [5]. This evidence appears to indicate a considerable distinctiveness in the $B x$ 's ability to evade or suppress host immune responses. Given that variant erythrocyte surface antigens (VESAs) encoded by ves genes $[8,36]$ have been implicated in immune evasion/modulation, the pathogenesis and/or the persistence of infections in the host, we focused our attention here on investigating the nature and extent of genes encoding these molecules in $B x$. Initially, the protein sequences homologous to those encoded by the ves genes of $B$. bovis, $B$. bigemina and $B$. divergens were identified. From the results, it became evident that VESAs in $B x$ were substantially distinct both in number and sequence from those of $B$. bovis, B. bigemina and $B$. divergens, such that they could not be classified in the same way as for their congeners. Therefore, we defined three distinct patterns that typify the 59 VESAs encoded in $B x$, and used these patterns as well as the VESA1_N domain to classify four distinct groups of VESAs (I-VI; Fig. 2): Specifically, group I proteins $(n=28)$ share the VESA1_N domain and have one or more additional patterns; group II proteins $(n=14)$ share a short domain and/or a pattern near the C-terminus (long proteins); group III proteins $(n=7)$ share a short domain and can have the pattern near the $\mathrm{N}$-terminus (long proteins); group VI proteins $(n=6)$ have no domain or pattern. Based on these results, it is evident that VESAs and their genes are highly labile or plastic in terms of genome 

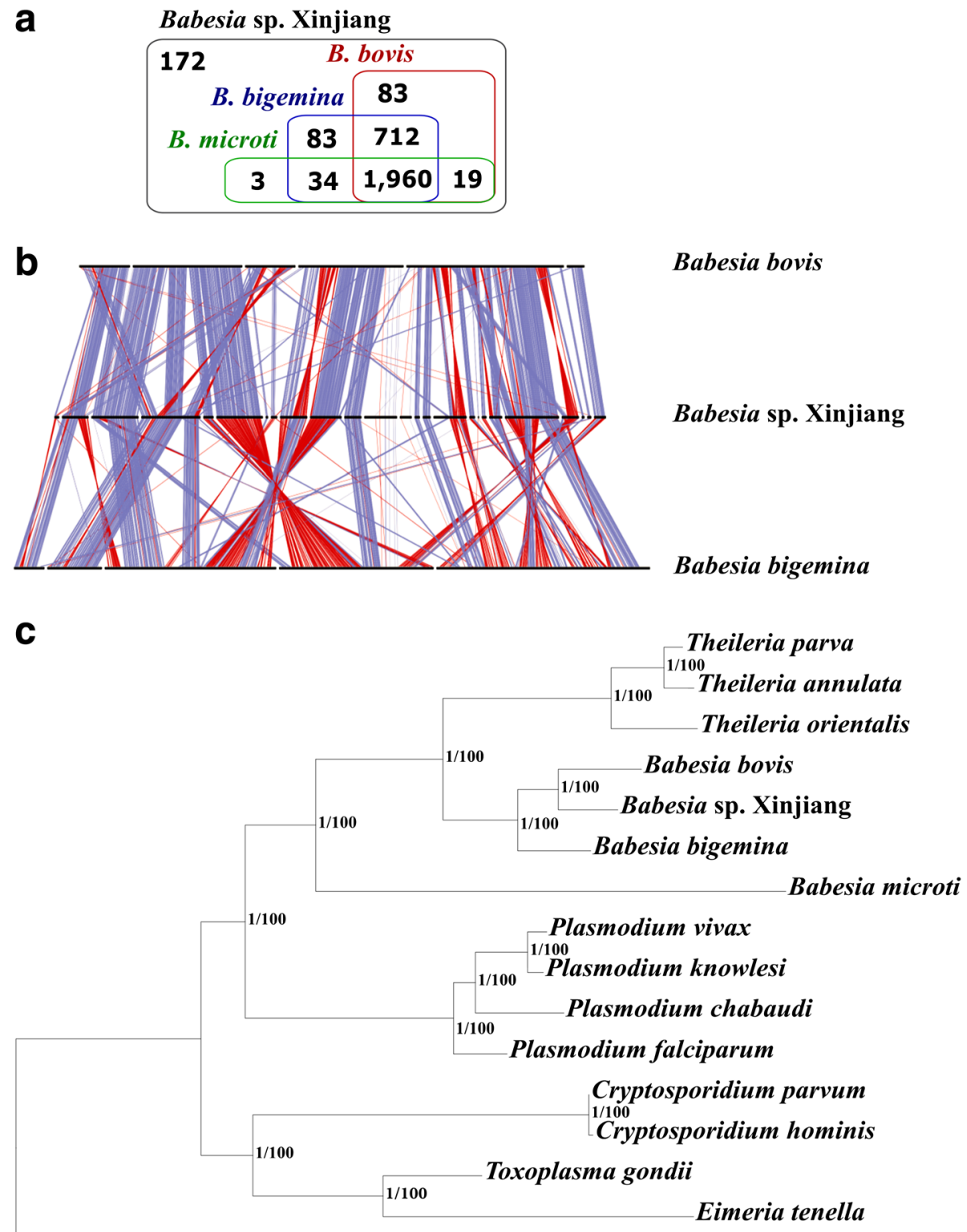

Tetrahymena thermophila

0.4

Fig. 1 a VENN diagram for the Babesia sp. Xinjiang (Bx) genes orthologous to those of B. bovis, B. bigemina and B. microti. Altogether, 1960 genes in $B x$ are shared with three other Babesia species, and 172 genes are unique to $B x$. b Single copy orthologous (SCO) genes ( $n=2136)$ among the scaffolds in the draft genomes of B. bovis, Bx and B. bigemina in the forward (blue) or reverse orientation (red). c Phylogenetic tree constructed from sequence data for SCOs $(n=326)$ shared among all apicomplexans for which proteomic data were available. The nodal support values were all 1.00 (posterior probability; pp) and $100 \%$ (bootstrap), indicated as '1/100'

repertoire and sequences, suggesting that the substantial divergence observed relates to frequent transposition to new genomic positions over time. Previous phylogenetic analyses of ves gene repertoires from various strains of $B$. bovis, $B$. bigemina and $B$. divergens from distinct geographical localities did not indicate strain-associated gene family expansions [8]; gene transposition appeared to be more frequent than evolution through amino acid substitution or gene duplication [8]. Moreover, most ves genes in $B x, B$. bovis and B. bigemina are not orthologous, even though they are relatively conserved in their position in the genome. Together with previous results [8], the present findings seem to support the proposal for a key role of recombination in Babesia, and that genomic architecture enables recombination to promote antigenic diversity and/or switching [66]. Consistent with var genes of 


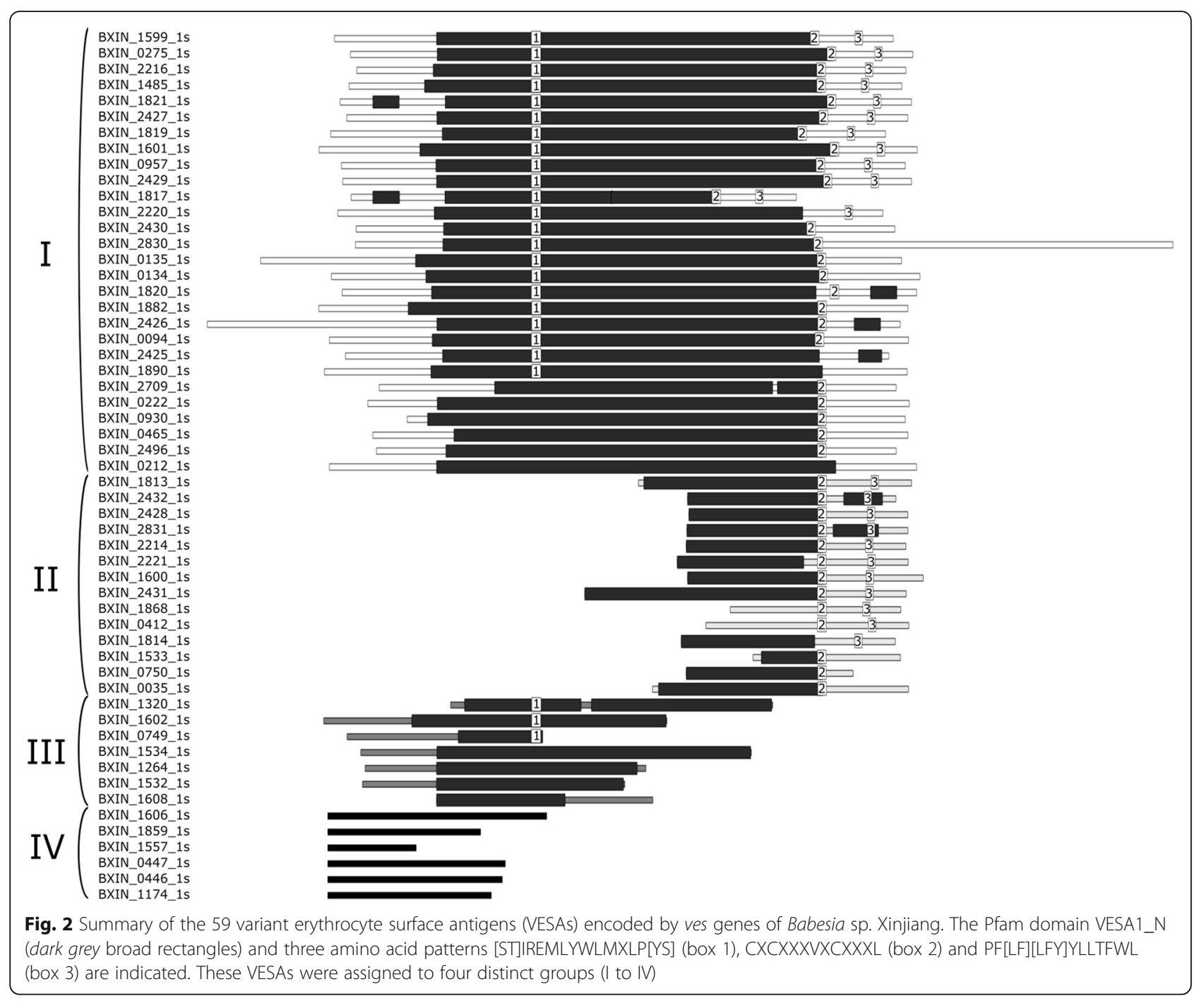

Plasmodium [67], rapid gene turnover, recombination and structural change appear to be responsible for ves gene diversity and complexity within and among Babesia species and their ability to induce disease and/or modulate or suppress host immune responses.

\section{Conclusions}

Although there have been some improvements in our understanding of the molecular biology of Babesia, progress has been relatively slow, as only a relatively small number of researchers around the world are investigating these apicomplexans. The genome of $B x$ provides a new and exciting resource for many future studies. Progress could proceed along many different lines.

One might be to complete the genome and sequence various species and strains of Babesia from small ruminants (sheep and goats) to chromosome-scale contiguity. Such an effort would resolve regions of tandem multigene families, which are often absent from assemblies of short read (Illumina) data sets [68], but that are central to understanding species- and/or strain-specific traits $[69,70]$. Another aspect could be to undertake detailed comparative analyses of the genome and transcriptome of $B x$ with other apicomplexans. Genomic comparisons could identify genes that are undergoing positive selection or gene family expansions or contractions in particular Babesia species, and may, therefore, provide insights into the evolution of gene families and their (possible) roles in virulence, pathogenicity and parasitism. Another avenue of investigation might be to explore the transcriptome of $B x$ in more detail and which genes or gene families are involved in parasitism. It would also be interesting to characterize stage-specific transcripts as well as ncRNAs to establish their contributions to a parasitic mode of existence. Moreover, the transcriptome of $B x$ could be used to model the parasite's metabolism (cf. [71]), which could be of considerable value if extended to other Babesia species. 
Clearly, there are many fundamental areas to tackle, to elucidate the biology of $B x$ and its relatives. In our opinion, a focus on molecular aspects of virulence, pathogenesis of disease, immune evasion or suppression as well as gene function would be particularly interesting, and could guide the discovery of new intervention strategies. With the availability of in vitro cultures for the maintenance and propagation of selected taxa, such as $B x$ [9] and $B$. bovis [8], there is now excellent potential to accelerate research of $B x$, and gain a deep understanding of its fundamental molecular biology and its differences from $B$. bovis. The ability to stably and transiently transform $B$. bovis, and genetically manipulate its genome [72-76] raises some prospect for developing a functional genomic platform for $B x$. Having such a platform in place would enable systems biological investigations using complementary genomic, transcriptomic and proteomic tools. It might also underpin applied research focused on developing new interventions, such as antiBabesia drugs or vaccines.

\section{Additional file}

Additional file 1: KEGG pathways for Babesia sp. Xinjiang. (XLSX 38 kb)

\section{Abbreviations}

BUSCO: Benchmarking Universal Single Copy Orthologs; Bx: Babesia sp. Xinjiang; KEGG: Kyoto Encyclopedia of Genes and Genomes; RNA-seq: RNAsequencing; SCO: Single Copy Ortholog; ves: Variant erythrocyte surface antigen gene; VESA: Variant Erythrocyte Surface Antigens (VESAs)

\section{Acknowledgements}

We thank BGI-Shenzhen for sequencing service.

\section{Funding}

This project was partially funded by the the PiroVac (KBBE-3-245145; H.Y), NSFC (31072130; G.G.), ASTIP, FRIP (2014ZL010), CAAS (H.Y.) and NBCIS (CARS-38; H.Y.) of China and the Australian Research Council (ARC) (R.B.G.); it was also supported by a Victorian Life Sciences Computation Initiative (VLSCl) grant (VR0007; R.B.G.) on its Peak Computing Facility at the University of Melbourne, an initiative of the Victorian Government. Other support from the State Key Laboratory of Veterinary Etiological Biology Project, China (SKLVEB2008ZZKT019; H.Y, the Australian Academy of Science, Alexander von Humboldt Foundation and Melbourne Water Corporation is gratefully acknowledged.

\section{Availability of data and material}

Accession codes: This whole genome shotgun project has been deposited in the NCBI BioProject database with accession code PRJNA329017 (BioSample: SAMN05389972). The project includes: the genome assembly under accession code MBFZ00000000; raw Illumina read sets for the genomic DNAs under accession codes SRR3923815, SRR3923816 and SRR3923817; raw reads for RNA-seq under accession code SRR3923818. The version of the genome described in this paper is MBFZ01000000.

\section{Authors' contributions}

GG, JL, YL, ZL and HY prepared merozoites and nucleic acids for sequencing and arranged Illumina sequencing. PKK and RBG planned the study. PKK undertook all bioinformatics analyses, with support from NDY, RBG and PKK wrote the manuscript with inputs from NDY, GG and comments from the other authors (AVK and TW). HY and RBG raised the funds for the project. All authors read and approved the final version of the manuscript.

\section{Competing interests}

The authors declare that they have no competing interests.

Consent for publication

Not applicable.

Ethics approval and consent to participate

Animal experimentation was approved (permit SYXK2010-0001) by the Science and Technology Department of Gansu province, China.

Received: 7 September 2016 Accepted: 12 October 2016

Published online: 27 October 2016

\section{References}

1. Bock R, Jackson L, De Vos A, Jorgensen W. Babesiosis of cattle. Parasitology. 2004:129:S247-S69.

2. Friedhoff K. Tick-borne diseases of sheep and goats caused by Babesia, Theileria or Anaplasma spp. Parassitologia. 1997;39:99-109.

3. Uilenberg G. Babesia - a historical overview. Vet Parasitol. 2006;138:3-10.

4. Cooke BM, Mohandas N, Cowman AF, Coppel RL. Cellular adhesive phenomena in apicomplexan parasites of red blood cells. Vet Parasitol. 2005;132:273-95.

5. Gohil S, Herrmann S, Günther S, Cooke BM. Bovine babesiosis in the 21st century: advances in biology and functional genomics. Int J Parasitol. 2013; 43:125-32.

6. Guan G, Ma M, Moreau E, Liu J, Lu B, Bai Q, et al. A new ovine Babesia species transmitted by Hyalomma anatolicum anatolicum. Exp Parasitol. 2009:122:261-67.

7. Guan $\mathrm{G}$, Yin $\mathrm{H}$, Luo J, Lu W, Zhang $\mathrm{Q}, \mathrm{Ma}$ M, et al. Isolation of a large ovine Babesia sp. Chin J Vet Sci Technol. 2001;31:35-6.

8. Jackson AP, Otto TD, Darby A, Ramaprasad A, Xia D, Echaide IE, et al. The evolutionary dynamics of variant antigen genes in Babesia reveal a history of genomic innovation underlying host-parasite interaction. Nucleic Acids Res. 2014;42:7113-31.

9. Guan G, Ma M, Liu A, Du P, Ren Q, Li Y, et al. Continuous in vitro cultivation of a recently identified Babesia that infects small ruminants in China. Vet Parasitol. 2012;187:371-78.

10. Guan G, Moreau E, Liu J, Ma M, Rogniaux H, Liu A, et al. BQP35 is a novel member of the intrinsically unstructured protein (IUP) family which is a potential antigen for the sero-diagnosis of Babesia sp. BQ1 (Lintan) infection. Vet Parasitol. 2012;187:421-30.

11. Bolger AM, Lohse $M$, Usadel B. Trimmomatic: a flexible trimmer for Illumina sequence data. Bioinformatics. 2014:30:2114-20.

12. Brown $C T$, Howe $A$, Zhang $Q$, Pyrkosz $A B$, Brom TH. A reference-free algorithm for computational normalization of shotgun sequencing data. NASA ADS. 2012;arXiv:1203.4802 [q-bio.GN].

13. Smit AFA, Robert $\mathrm{H}$, Kas A, Siegel A, Gish W, Price A, et al. RepeatModeler. In., 1.0.5 edn. http://www.repeatmasker.org: Institute of Systems Biology; 2011.

14. Bao Z, Eddy SR. Automated de novo identification of repeat sequence families in sequenced genomes. Genome Res. 2002;12:1269-76.

15. Price AL, Jones NC, Pevzner PA. De novo identification of repeat families in large genomes. Bioinformatics. 2005;21 Suppl 1:i351-58.

16. Benson G. Tandem repeats finder: a program to analyze DNA sequences. Nucleic Acids Res. 1999;27:573-80.

17. Xu Z, Wang H. LTR_FINDER: an efficient tool for the prediction of full-length LTR retrotransposons. Nucleic Acids Res. 2007;35:W265-W68.

18. Smit AFA, Hubley R, Green P: RepeatMasker. In: http://www.repeatmasker. org: Institute of Systems Biology; 1996-2010.

19. Jurka J, Kapitonov W, Pavlicek A, Klonowski P, Kohany O, Walichiewicz J. Repbase update, a database of eukaryotic repetitive elements. Cytogenet Genome Res. 2005:110:462-67.

20. Bankevich A, Nurk S, Antipov D, Gurevich AA, Dvorkin M, Kulikov AS, et al. SPAdes: a new genome assembler and its applications to single cell sequencing. J Comput Biol. 2012:19:455-77.

21. Boetzer M, Henkel CV, Jansen HJ, Butler D, Pirovano W. Scaffolding preassembled contigs using SSPACE. Bioinformatics. 2011;27:578-79.

22. Holt C, Yandell M. MAKER2: an annotation pipeline and genome-database management tool for second-generation genome projects. BMC Bioinformatics. 2011;12:491. 
23. Stanke M, Waack S. Gene prediction with a hidden Markov model and a new intron submodel. Bioinformatics. 2003;19 Suppl 2:ii215-25.

24. Borodovsky M, Lomsadze A. Eukaryotic gene prediction using GeneMark.hmm-E and GeneMark-ES. Curr Protoc Bioinformatics. 2011; Chapter 4: Unit 4.6 1-10.

25. Korf I. Gene finding in novel genomes. BMC Bioinformatics. 2004;5:59.

26. Kim D, Pertea G, Trapnell C, Pimentel H, Kelley R, Salzberg SL. TopHat2: accurate alignment of transcriptomes in the presence of insertions, deletions and gene fusions. Genome Biol. 2013;14:R36.

27. Trapnell C, Hendrickson DG, Sauvageau M, Goff L, Rinn JL, Pachter L. Differential analysis of gene regulation at transcript resolution with RNA-seq. Nat Biotechnol. 2013;31:46-53.

28. Haas BJ, Papanicolaou A, Yassour M, Grabherr M, Blood PD, Bowden J, et al. De novo transcript sequence reconstruction from RNA-seq using the Trinity platform for reference generation and analysis. Nat Protoc. 2013;8:1494-512.

29. Pruitt KD, Tatusova T, Maglott DR. NCBI reference sequence (RefSeq): a curated non-redundant sequence database of genomes, transcripts and proteins. Nucleic Acids Res. 2005:33:D501-4.

30. Haas BJ, Salzberg SL, Zhu W, Pertea M, Allen JE, Orvis J, et al. Automated eukaryotic gene structure annotation using EVidenceModeler and the program to assemble spliced alignments. Genome Biol. 2008;9:1.

31. Lowe TM, Eddy SR. tRNAscan-SE: a program for improved detection of transfer RNA genes in genomic sequence. Nucleic Acids Res. 1997;25:955-64.

32. Simão FA, Waterhouse RM, loannidis $P$, Kriventseva EV, Zdobnov EM. BUSCO: assessing genome assembly and annotation completeness with single-copy orthologs. Bioinformatics. 2015;31(19):3210-2.

33. Hunter $\mathrm{S}$, Jones $\mathrm{P}$, Mitchell A, Apweiler R, Attwood TK, Bateman A, et al. InterPro in 2011: new developments in the family and domain prediction database. Nucleic Acids Res. 2012;40:D306-12.

34. Altschul SF, Madden TL, Schaffer AA, Zhang J, Zhang Z, Miller W, et al. Gapped BLAST and PSI-BLAST: a new generation of protein database search programs. Nucleic Acids Res. 1997;25:3389-402.

35. Camacho C, Coulouris G, Avagyan V, Ma N, Papadopoulos J, Bealer K, et al. BLAST+: architecture and applications. BMC Bioinformatics. 2009;10:421.

36. Brayton KA, Lau AO, Herndon DR, Hannick L, Kappmeyer LS, Berens SJ, et al. Genome sequence of Babesia bovis and comparative analysis of apicomplexan hemoprotozoa. PLoS Pathog. 2007;3:e148.

37. Magrane M, Consortium U. UniProt knowledgebase: a hub of integrated protein data. Database (Oxford). 2011;2011:bar009.

38. Kanehisa M, Goto S, Sato Y, Furumichi M, Tanabe M. KEGG for integration and interpretation of large-scale molecular datasets. Nucleic Acids Res. 2012; 40:D109-D14.

39. Pruitt KD, Tatusova T, Klimke W, Maglott DR. NCBI reference sequences: current status, policy and new initiatives. Nucleic Acids Res. 2009;37:D32-6.

40. Petersen TN, Brunak S, von Heijne G, Nielsen H. SignalP 4.0: discriminating signal peptides from transmembrane regions. Nat Methods. 2011;8:785-6.

41. Kall L, Krogh A, Sonnhammer EL. A combined transmembrane topology and signal peptide prediction method. J Mol Biol. 2004;338:1027-36.

42. Hall B, DeRego T, Geib S: GAG: the genome annotation generator. In., 1.0 edn. http://genomeannotation.github.io/GAG; 2014.

43. Van Dongen S. Graph clustering by flow simulation. The Netherlands: University of Utrecht; 2000.

44. Li L, Stoeckert Jr CJ, Roos DS. OrthoMCL: identification of ortholog groups for eukaryotic genomes. Genome Res. 2003:13:2178-89.

45. Katoh K, Kuma K, Toh H, Miyata T. MAFFT version 5: improvement in accuracy of multiple sequence alignment. Nucleic Acids Res. 2005:33:511-8.

46. Ronquist F, Huelsenbeck JP. MrBayes 3: Bayesian phylogenetic inference under mixed models. Bioinformatics. 2003;19:1572-74.

47. Altekar G, Dwarkadas S, Huelsenbeck JP, Ronquist F. Parallel metropolis coupled Markov chain Monte Carlo for Bayesian phylogenetic inference. Bioinformatics. 2004:20:407-15.

48. Stamatakis A, Ludwig T, Meier H. RAxML-III: a fast program for maximum likelihood-based inference of large phylogenetic trees. Bioinformatics. 2005; 21:456-63.

49. Darriba D, Taboada GL, Doallo R, Posada D. ProtTest 3: fast selection of bestfit models of protein evolution. Bioinformatics. 2011;27:1164-5.

50. Whelan S, Goldman N. A general empirical model of protein evolution derived from multiple protein families using a maximum-likelihood approach. Mol Biol Evol. 2001;18:691-99.

51. Yang Z. Maximum likelihood phylogenetic estimation from DNA sequences with variable rates over sites: approximate methods. J Mol Evol. 1994;39:306-14.
52. Yang ZH. Maximum-likelihood-estimation of phylogeny from DNA sequences when substitution rates differ over sites. Mol Biol Evol. 1993;10: 1396-401.

53. Metropolis $\mathrm{N}$, Rosenbluth AW, Rosenbluth $\mathrm{AH}$, Teller $\mathrm{H}$, Teller E. Equations of state calculations by fast computing machines. J Chem Phys. 1953;21:1087-91.

54. Hastings WK. Monte-Carlo sampling methods using Markov chains and their applications. Biometrika. 1970;57:97-109.

55. Geyer CJ. Markov-chain Monte-Carlo maximum-likelihood. Comp Sci Stat. 1991:156-63.

56. Jones DT, Taylor WR, Thornton JM. The rapid generation of mutation data matrices from protein sequences. Comput Appl Biosci. 1992;8:275-82.

57. Jünger M, Mutzel P. 2-Layer straightline crossing minimization: Performance of exact and heuristic algorithms. J Graph Algorithms Appl. 1997:1:1-25.

58. Benson SJ, Ye Y. DSDP5: Software for semidefinite programming. Argonne: Mathematics and Computer Science Division, Argonne National Laboratory; 2005. Tech Rep ANL/MCS-P1289-0905.

59. Jonassen I, Collins JF, Higgins DG. Finding flexible patterns in unaligned protein sequences. Protein Sci. 1995:4:1587-95.

60. Eddy SR. Profile hidden Markov models. Bioinformatics. 1998;14:755-63.

61. Finn RD, Coggill P, Eberhardt RY, Eddy SR, Mistry J, Mitchell AL, et al. The Pfam protein families database: towards a more sustainable future. Nucleic Acids Res. 2016:44:D279-D85.

62. Cornillot E, Hadj-Kaddour K, Dassouli A, Noel B, Ranwez V, Vacherie B, et al. Sequencing of the smallest apicomplexan genome from the human pathogen Babesia microti. Nucleic Acids Res. 2012;40:9102-14.

63. Nakajima R, Tsuji M, Oda K, Zamoto-Niikura A, Wei Q, Kawabuchi-Kurata T, et al. Babesia microti-group parasites compared phylogenetically by complete sequencing of the CCT. ETA. gene in 36 isolates. J Vet Med Sci. 2009;71:55-68.

64. Cornillot E, Dassouli A, Garg A, Pachikara N, Randazzo S, Depoix D, et al. Whole genome mapping and re-organization of the nuclear and mitochondrial genomes of Babesia microti isolates. PLoS One. 2013;8: e72657.

65. Tian Z-C, Liu G-Y, Yin H, Luo J-X, Guan G-Q, Luo J, et al. RPS8 - a new informative DNA marker for phylogeny of Babesia and Theileria parasites in China. PLoS One. 2013;8:e79860.

66. Kirkman LA, Deitsch KW. Antigenic variation and the generation of diversity in malaria parasites. Curr Opin Microbiol. 2012;15:456-62.

67. Kraemer SM, Smith JD. Evidence for the importance of genetic structuring to the structural and functional specialization of the Plasmodium falciparum var gene family. Mol Microbiol. 2003;50:1527-38.

68. Alkan C, Sajjadian S, Eichler EE. Limitations of next-generation genome sequence assembly. Nat Methods. 2011;8:61-5.

69. Kondrashov FA. Gene duplication as a mechanism of genomic adaptation to a changing environment. Proc Roy Soc Lond B Bio. 2012;279:5048-57.

70. Qian W, Zhang J. Genomic evidence for adaptation by gene duplication. Genome Res. 2014;24:1356-62.

71. O'Brien EJ, Monk JM, Palsson BO. Using genome-scale models to predict biological capabilities. Cell. 2015;161:971-87.

72. Suarez CE, McElwain TF. Transient transfection of purified Babesia bovis merozoites. Exp Parasitol. 2008;118:498-504.

73. Suarez CE, McElwain TF. Stable expression of a GFP-BSD fusion protein in Babesia bovis merozoites. Int J Parasitol. 2009:39:289-97.

74. Suarez CE, McElwain TF. Transfection systems for Babesia bovis: a review of methods for the transient and stable expression of exogenous genes. Vet Parasitol. 2010;167:205-15.

75. Suarez CE, Norimine J, Lacy P, McElwain TF. Characterization and gene expression of Babesia bovis elongation factor-1a. Int J Parasitol. 2006;36:965-73.

76. Suarez CE, Palmer GH, LeRoith T, Florin-Christensen M, Crabb B, McElwain TF. Intergenic regions in the rhoptry associated protein-1 (rap-1) locus promote exogenous gene expression in Babesia bovis. Int J Parasitol. 2004; 34:1177-84. 